\title{
Probing additional dimensions in the universe with neutron experiments
}

\author{
Alejandro Frank ${ }^{\mathrm{a}, \mathrm{b}}$, Piet Van Isacker ${ }^{\mathrm{c}}$, Joaquín Gómez-Camacho ${ }^{\mathrm{d}}$ \\ a Instituto de Ciencias Nucleares, Universidad Nacional Autónoma de México, Apartado Postal 70-543, 04510 México, D.F., Mexico \\ ${ }^{\mathrm{b}}$ Centro de Ciencias Físicas, Universidad Nacional Autónoma de México, Apartado Postal 139-B, 62251 Cuernavaca, Morelos, Mexico \\ c GANIL, B.P. 55027, F-14076 Caen Cedex 5, France \\ ${ }^{\mathrm{d}}$ Departamento de Física Atómica, Nuclear y Molecular, Facultad de Física, Universidad de Sevilla, Sevilla, Spain
}

Received 12 May 2003; received in revised form 17 November 2003; accepted 9 December 2003

Editor: W. Haxton

\begin{abstract}
We carry out a simple analysis of $(n+3)$-dimensional gravity in the context of recent work on 'large' supplementary dimensions and deduce a formula for the expected compactification radius for the $n$ additional dimensions in the universe, as a function of the Planck and the electro-weak scales. We argue that the correspondingly modified gravitational force gives rise to effects that might be within the detection range of dedicated neutron experiments. A scattering analysis of the corresponding modified gravitational forces suggests that slow neutron scattering off atomic nuclei with null spin may provide an experimental test for these ideas.
\end{abstract}

(C) 2004 Published by Elsevier B.V. Open access under CC BY license.

The study of gravity at short range has recently been the subject of numerous theoretical and experimental investigations, sparked by the proposal by Arkani-Hamed, Dimoupoulos, and Dvali (ADD) [1] that gravity may depart from Newton's inverse square law at scales which could be as large as a millimeter, a scenario that was subsequently shown to be consistent with string theory [2]. Diverse experimental groups have built refined versions of torsion balance experiments and other ingenious designs to test gravity at submillimeter ranges [3]. On the theoretical front, hundreds of papers have been written, ranging from alternative formulations of the large extra-

E-mail address: isacker@ganil.fr (P. Van Isacker). dimensions framework (LED) [4], to the study of astrophysical constraints and the expected experimental consequences in future high-energy collider experiments to black-hole production and the effect of LED on fundamental symmetries. But perhaps the most remarkable result to date is the fact that no known physical constraints have as yet falsified the LED theories. From the experimental point of view the new measurements have confirmed the validity of Newton's law to about $0.1 \mathrm{~mm}$. Although such efforts are of great value, it can hardly be expected that this kind of experiments can lower this limit significantly.

We show in this Letter that a simple analysis of $N$ dimensional $(N \mathrm{D})$ gravity leads to compactification lengths in close agreement with the more sophisticated calculations of general relativity and string theory [5]. 
Following this cue, we then present calculations that suggest that the effects of $N D$ forces, although very small, might for particular values of $N$ fall within the detection range of dedicated neutron experiments.

In their papers, ADD conjecture the existence of two or more additional dimensions in which gravity, but not the strong or the electro-weak forces, might be acting, diluting itself by spreading its lines of force into these extra dimensions. Essentially, this would explain its apparent weakness [1]. The proposal arises from a bold modification of pre-existing many-dimensional string theories and the more recent $\mathrm{M}$-theories ( $\mathrm{M}$ for membrane) which encompass the former [6]. In these theories only gravitons are able to traverse the extra dimensions, whereas other particles are fixed to our observable 3D world, since the former are described as closed strings free to wander while the latter are open strings with their ends fixed to our 'brane'. Additional dimensions are 'compactified', i.e., they are closed on themselves with a characteristic radius of compactification $R_{\mathrm{c}}$ (which for simplicity is assumed to be the same for all additional dimensions). For ranges smaller than $R_{\mathrm{c}}$, we thus expect a modified gravitational force. The basic question is then: why should $R_{\mathrm{c}}$ be large compared with M-theory's original Planck scale of $\sim 10^{-35} \mathrm{~m}$ ? The answer rests on the empirical fact that gravitation has never been measured below about $10^{-4} \mathrm{~m}$ and more significantly, on the profound theoretical implications that 'large' extra dimensions would have on the solution of the hierarchy problem whose origin can be traced to the huge difference in strength observed between gravity on the one hand and the other forces described by the standard model [7]. In this scenario, instead of catching up with the other forces at Planck's length scale, the $N \mathrm{D}$ gravitational force actually joins the other interactions at a distance about $10^{16}$ times larger, namely, at the electro-weak unification scale of $\sim 10^{-19} \mathrm{~m}$. As will be shown below, this conjecture fixes the strength of the $N D$ gravitational force and the value of $R_{\mathrm{c}}$. The most important consequence of the LED hypothesis is the possible transition from the entirely Platonic, inaccessible situation posed by the Planck-scale compactification, to one where it is conceivable that experimental measurements may actually test these ideas. The experiments envisioned to date are predominantly of two kinds. The ones involving submillimeter torsionbalance experiments [3] mentioned above, and high- energy collider experiments in the $\mathrm{TeV}$ energy region, where diverse theoretical predictions exist for the indirect observation of additional dimensions, such as the occurrence of missing energy carried away by undetected gravitons [8]. The question arises as to whether other experiments can be designed to probe gravity at very short ranges. It has been suggested, for example, that the increasing precision of experimental tests of the Casimir effect may be used to probe new forces at micrometer distances [9]. Here we explore the possible effects of $N D$ gravity in experiments with neutrons.

We start in the spirit of ADD [1] by carrying out a classical analysis of gravity in $N=n+3$ dimensions. If space would have $n+3$ (extended) dimensions, Gauss' law implies that the force of gravity would be of the form

$F_{n}=-\frac{m_{1} m_{2} G_{n}}{r^{n+2}}$

where $G_{n}$ is a constant which reduces to Newton's gravitational constant $G$ for $n=0$. As explained above, we shall follow M-theory and assume that the masses, as well as all other forces remain in 3D space and only the gravitational force field leaks into the additional dimensions. Even after compactification of these additional dimensions, formula (1) should be correct for $r \leqslant R_{\mathrm{c}}$.

In general, one should assume a soft transition from $N D$ gravity to $3 \mathrm{D}$ gravity. We shall instead follow a simpler procedure and consider a sudden transition from $N D$ to $3 \mathrm{D}$ using Eq. (1). We first impose the equality of forces at the compactification length $R_{\mathrm{c}}$, $F_{n}\left(R_{\mathrm{c}}\right)=F_{0}\left(R_{\mathrm{c}}\right)$, which implies that

$F_{n}=-\frac{m_{1} m_{2} G R_{\mathrm{c}}^{n}}{r^{n+2}}$.

We then implement the ADD conjecture that the $N D$ force at the electro-weak length $R_{\mathrm{e}}$ is as strong as the $3 \mathrm{D}$ force at the Planck length $R_{\mathrm{P}}, F_{n}\left(R_{\mathrm{e}}\right)=F_{0}\left(R_{\mathrm{P}}\right)$. This leads to the desired equation

$$
\left(\frac{R_{\mathrm{c}}}{R_{\mathrm{e}}}\right)^{n}=\left(\frac{R_{\mathrm{e}}}{R_{\mathrm{P}}}\right)^{2}
$$

which expresses the compactification length $R_{\mathrm{c}}$ as a function of the two fundamental scales $R_{\mathrm{e}}$ and $R_{\mathrm{P}}$. These lengths are defined as

$$
R_{\mathrm{P}}=\sqrt{\hbar G / c^{3}}, \quad R_{\mathrm{e}}=\sqrt{G_{\mathrm{F}} / \hbar c},
$$


where $G$ and $G_{\mathrm{F}}$ are Newton's and Fermi's constants, respectively, the latter defined by [7]

$G_{\mathrm{F}}=\frac{\sqrt{2} \hbar^{2} g^{2}}{8 c^{2} M_{W}^{2}}=\left(1.166 \times 10^{-5} \mathrm{GeV}^{-2}\right)(\hbar c)^{3}$,

where $M_{W}$ is the mass of the $W_{ \pm}$bosons and $g \times$ $\sin \theta_{\mathrm{W}}=e$, with $\theta_{\mathrm{W}}$ the Weinberg angle. We use cgs units throughout. We then find that formula (3) can be written in the form:

$\left(\frac{R_{\mathrm{c}}}{R_{\mathrm{e}}}\right)^{n}=\frac{c^{2}}{\hbar^{2}} \frac{G_{\mathrm{F}}}{G}$.

Note that this expression relates the number of additional dimensions $n$, the radius of compactification $R_{\mathrm{c}}$, and the electro-weak scale $R_{\mathrm{e}}$, to the ratio of two fundamental numbers in nature: Fermi's and Newton's constants, with $c^{2} / \hbar^{2}$ as a proportionality constant.

To have an indication of whether formula (4) is robust, we remark that a similar result can be obtained using a different argument. We require that at the electroweak length scale the $N \mathrm{D}$ force be comparable to the electromagnetic interaction. We may assume that at distances of order $R_{\mathrm{e}}$ most particles are ultrarelativistic and thus that their masses should be of order $\hbar / R_{\mathrm{e}} c$. Equating the electromagnetic interaction to the gravitational force between two masses of this order we arrive at

$\frac{\hbar^{2}}{R_{\mathrm{e}}^{2} c^{2}} \frac{G R_{\mathrm{c}}^{n}}{R_{\mathrm{e}}^{n+2}}=\frac{e^{2}}{R_{\mathrm{e}}^{2}}$.

This leads to the relation

$\left(\frac{R_{\mathrm{c}}}{R_{\mathrm{e}}}\right)^{n}=\alpha \frac{c^{2}}{\hbar^{2}} \frac{G_{\mathrm{F}}}{G}=\alpha\left(\frac{R_{\mathrm{e}}}{R_{\mathrm{P}}}\right)^{2}$, where $\alpha$ is the fine-structure constant which is about $1 / 128$ at the electroweak scale $R_{\mathrm{e}}$. Values for $R_{\mathrm{c}}$ obtained with expressions (4) and (6) are compared in Table 1.

The present model can be interpreted as follows: the Fermi constant is related in the standard electroweak theory to the value of the scalar field that produces the Higgs mechanism of spontaneous symmetry breaking. The Higgs boson, which is yet to be discovered, corresponds to the excitation mode of this scalar field. However, in this model $G_{\mathrm{F}}$ also represents the intensity of the gravitational field in $N$ dimensions. Thus, this model suggests the exciting possibility that the scalar field which is needed in the standard model may just be the gravitational field which is wrapped out into the additional dimensions [10]. The very small Newton constant $G$ gives the residual value of the gravitational field which spills out into the usual three dimensions, beyond the radius of compactification $R_{\mathrm{c}}$.

Carrying further this line of thinking, we can consider that, for distances larger than $R_{\mathrm{c}}$, the gauge forces live in the 3-dimensional brane, and so does the normal, very weak gravity. Between $R_{\mathrm{c}}$ and $R_{\mathrm{e}}$, the gauge forces still live in three dimensions, while gravity lives in $N$ dimensions, increasing its strength as the distance decreases, so that for $r=R_{\mathrm{e}}$ is becomes comparable to the electro-weak force. At this point, and due to a yet unknown mechanism, gravity generates the scalar field that couples to the gauge field giving rise to the Higgs mechanism.

In Table 1 we display both values of $R_{\mathrm{c}}$ as a function of $n$, which turn out to be close to the ones evaluated by other means. Note that $n=1$ can be readily discarded since it leads to a value of $R_{\mathrm{c}}$ larger

Table 1

Estimates of various lengths (in fm), energies (in $\mathrm{MeV}$ ), and phase shifts (in rad) as a function of the number of extra dimensions $n$

\begin{tabular}{llllllll}
\hline$n$ & 1 & 2 & 3 & 4 & 5 & 6 & 7 \\
\hline$R_{\mathrm{c}}$ (Eq. (3)) & $1.2 \times 10^{30}$ & $2.8 \times 10^{13}$ & $8.1 \times 10^{7}$ & $1.4 \times 10^{5}$ & $3.0 \times 10^{3}$ & $2.3 \times 10^{2}$ & 38 \\
$R_{\mathrm{c}}$ (Eq. (6)) & $9.3 \times 10^{27}$ & $2.5 \times 10^{12}$ & $1.6 \times 10^{7}$ & $4.2 \times 10^{4}$ & $1.1 \times 10^{3}$ & $1.0 \times 10^{2}$ & 19 \\
$V_{n}(r=30)$ & $1.6 \times 10^{-7}$ & $2.4 \times 10^{-12}$ & $4.0 \times 10^{-17}$ & $7.1 \times 10^{-22}$ & $1.3 \times 10^{-26}$ & $2.6 \times 10^{-31}$ & $5.1 \times 10^{-36}$ \\
$R_{=}$ & 18 & 25 & 32 & 39 & 47 & 54 & 62 \\
$V_{n}\left(R_{=}\right)$ & $4.4 \times 10^{-7}$ & $4.1 \times 10^{-12}$ & $3.1 \times 10^{-17}$ & $1.9 \times 10^{-22}$ & $9.0 \times 10^{-28}$ & $4.2 \times 10^{-33}$ & $1.5 \times 10^{-38}$ \\
$E_{\text {min }}$ & - & - & $2.3 \times 10^{-13}$ & $7.9 \times 10^{-8}$ & $1.7 \times 10^{-4}$ & $2.7 \times 10^{-2}$ & 1.1 \\
$E_{\text {opt }}$ & 4.6 & 2.3 & 1.5 & 0.98 & 0.68 & 0.51 & 0.39 \\
$\phi_{\text {opt }}$ & $7.1 \times 10^{-8}$ & $1.2 \times 10^{-12}$ & $1.6 \times 10^{-17}$ & $2.1 \times 10^{-22}$ & $4.9 \times 10^{-28}$ & $5.6 \times 10^{-33}$ & $1.2 \times 10^{-38}$ \\
\hline
\end{tabular}


than the size of the solar system and hence to unstable planetary orbits. For $n=2$ we find $R_{\mathrm{c}} \sim \mathrm{mm}$ or $\mathrm{cm}$. Deviations from Newton's law at this scale seem to be discarded by experiment. Nevertheles, it should be noted that our analysis can only be expected to give rough estimates with considerable uncertainties in the prediction of $R_{\mathrm{c}}$. It is the range below $1 \mathrm{~mm}$, which is very difficult to explore with macroscopic gravity experiments, that we would like to investigate by means of neutrons. We should note that even if the LED hypothesis turns out to be wrong, it is still an interesting question to analyze whether neutron experiments can unveil deviations from Newton's law at short distances.

The physics of slow neutrons has undergone significant evolution in the last decades. Neutrons have become a standard probe for nuclear physics experiments as well as for other areas including the study and dynamics of condensed matter [11]. Pulsed neutron beams can currently be generated with very precise energies and polarizations and neutron detectors achieve very high efficiencies. Delicate experiments with thermal neutrons have recently demonstrated the quantization of their energies when subject to Earth's gravitational field [12]. In order to attempt neutron $N D$ gravity experiments at short ranges we face two problems from the outset. The more obvious one is the strong nuclear force, present at range scales of the order of $10^{-15} \mathrm{~m}$. A second, less obvious problem is that even for a spin-zero target nucleus, a neutron approaching it with speed $v$ feels a magnetic field $\vec{B}=\frac{1}{2 c} \vec{E} \times \vec{v}$ in its rest frame, where $\vec{E}$ is the nuclear electric field due to its charge $Z$. A long-range electromagnetic interaction (the Schwinger effect [13]) of strength $\vec{\mu} \cdot \vec{B}$ results, where $\vec{\mu}$ is the neutron magnetic moment. Neutrons have been proposed primarily to avoid direct competition with the much stronger electromagnetic interaction. We see that there is a residual, relativistic effect which needs to be dealt with. To minimize this potentially competing interaction slow neutrons are required, possibly polarized in the incident direction. (Note that the effect averages out to zero for unpolarized projectiles.) Very slow neutrons will suffer essentially pure $s$-wave nuclear scattering, while the longer range $N D$ gravitational force can in principle produce scattering of higher $l$-waves. The main question is whether interference effects between nuclear and gravitational scattering can be detected, in a fashion similar to the observed interference effects between nuclear and electromagnetic forces in heavyion reactions which give rise to 'rainbow' scattering and other such phenomena [14].

The potential that produces the modified gravitational force for distances $r$ below $R_{\mathrm{c}}$ can be written as

$V_{n}(r)=\frac{m_{1} m_{2} G R_{\mathrm{c}}^{n}}{(n+1) r^{n+1}}$.

Let us first consider the interaction of a neutron beam with a heavy nucleus such as ${ }^{208} \mathrm{~Pb}$. The constant $m_{1} m_{2} G$ is extremely small in this case giving rise to very small values of the potential energy at a typical distance of $r=30 \mathrm{fm}$ (see Table 1). We will discuss what are the optimal experimental conditions which could allow observation of this tiny effect in neutronscattering experiments and will consider what is the adequate energy and angular momentum so that the phase shift due to the gravitational force is as large as possible.

The nuclear potential can be parametrized with a Woods-Saxon shape, so that

$V_{\text {nucl }}(r)=\frac{V_{0}}{1+\exp (r-R) / a}$.

Reasonable parameters are $V_{0}=50 \mathrm{MeV}, R=1.2 \times$ $A^{1 / 3} \mathrm{fm}$, and $a=0.6 \mathrm{fm}$. This gives, for distances below $r=10 \mathrm{fm}$, values of the potential in the $\mathrm{MeV}$ range and any gravitational effect at that distance would be drowned by the uncertainties in the nuclear potential. Instead, one must probe distances at which the nuclear and gravitational potential are of the same order. In Table 1 we indicate the distances $R_{=}$at which the nuclear and gravitational potential are equal, as a function of $n$. For these calculations we have used the estimates for $R_{\mathrm{c}}$ from Eq. (4) although Eq. (6) leads to similar results. Any scattering observable that is affected by distances smaller than $R=$ will be contaminated by nuclear effects. We find that, if the number of additional dimensions is larger than $n=6$, then the gravitational force will be smaller than the nuclear force for any value of $r<R_{\mathrm{c}}$. For $n \leqslant 6$ there exists a range $R_{=}<r<R_{\mathrm{c}}$ in which the gravitational effects are larger than nuclear effects, and, at least in principle, might be measurable although small.

The scattering observables are also affected by the fact that the neutron has bound states in the 
nuclear potential generated by ${ }^{208} \mathrm{~Pb}$. From the shell structure of this nucleus one knows that the singleparticle potential supports bound states up to angular momentum $L=7$ (the $1 j_{15 / 2}$ orbital). Thus, the scattering of neutrons with $L \leqslant 7$ is affected by the nuclear potential, even if the scattering is very small, because the scattering wave functions have to be orthogonal to the bound states. Consequently, to obtain scattering observables free of nuclear contamination, we need to consider $L>7$.

In order to investigate gravitational effects, the energy of the neutron cannot be arbitrarily low. As one is investigating effects which occur at distances below the compactification length $R_{\mathrm{c}}$, the wave length of the neutron should be smaller than $R_{\mathrm{c}}$. More specifically, the momentum of the neutron should be such that

$p_{\mathrm{n}} R_{\mathrm{c}}>\left(L_{\min }+1 / 2\right) \hbar$,

where $L_{\min }=8$ in the example of ${ }^{208} \mathrm{~Pb}$. The corresponding minimum energies of the neutron,

$E_{\min }=\frac{p_{\mathrm{n}}^{2}}{2 m_{\mathrm{n}}}=\frac{\left(L_{\min }+1 / 2\right)^{2} \hbar^{2}}{2 m_{\mathrm{n}} R_{\mathrm{c}}^{2}}$,

are given in Table 1.

Estimates of the phase shifts due to the gravitational interaction can be found in the eikonal approximation where they are obtained for a given angular momentum in terms of the time integral of the potential along a straight line trajectory which has the same angular momentum:

$\phi(L, E) \simeq \frac{1}{\hbar} \int V\left(\sqrt{b^{2}+v^{2} t^{2}}\right) d t$,

$b=\frac{\hbar(L+1 / 2)}{m_{\mathrm{n}} v}$.

The time integral can be estimated by taking the potential at the point of closest approach $r=b$, multiplied by the approximate characteristic time $\tau=$ $b / v$ of interaction. In this way, we find

$\phi(L, E, n) \simeq \frac{m_{1} m_{2} G R_{\mathrm{c}}^{n}}{(n+1) b^{n} \hbar v}$.

From this expression we see that, in order to enhance the scattering effects of the gravitational force, one would need to have, in principle, small impact parameter and small velocity which are related through $b_{v m}=(L+1 / 2) \hbar$. To maximize the phase shift $\phi(L, E, n)$, the best choice is to take the minimum angular momentum $L_{\min }=8$ and the minimum impact parameter which corresponds to the distance $R_{=}$ at which the nuclear force may start to play a role. This gives the following optimal energy for scattering:

$E_{\mathrm{opt}}=\frac{\left(L_{\min }+1 / 2\right)^{2} \hbar^{2}}{2 m_{\mathrm{n}} R_{=}^{2}}$.

These energies are shown in Table 1 and are of the order of $1 \mathrm{MeV}$. At these energies the optimal phase shifts (for $L=L_{\min }$ ), also shown in Table 1, can be evaluated to give

$\phi_{\mathrm{opt}} \simeq \frac{V_{n}\left(R_{=}\right)}{2 E_{\mathrm{opt}}}$.

For $n>2$ the phase shift is extremely small and decreases as $n$ grows. However, we believe that the case of $n=2$ may be within reach of current dedicated scattering experiments. For this case, the elastic scattering amplitude produced by the modified gravitational force is given by the expression

$A_{g}(E, \theta)=\frac{i}{k} \sum_{L}(2 L+1) \phi(L, E, 2) P_{L}(\cos \theta)$,

where $\phi(L, E, 2)$ is given by Eq. (12). This amplitude turns out to be energy-independent and can be written in the closed form

$A_{g}(E, \theta)=i A_{g} f_{g}(\theta), \quad A_{g}=\frac{2 \hbar c\left(m_{n} m_{T} G_{F}\right)^{2}}{3\left(m_{n}+m_{T}\right)}$,
$f_{g}(\theta)=\sum_{L}(L+1 / 2)^{-1} P_{L}(\cos \theta)$,

where we have made use of Eq. (4). The modified gravitational amplitude can now be evaluated for the scattering of neutrons on ${ }^{208} \mathrm{~Pb}$, using Eq. (16) and we find $A_{g}=0.298 \times 10^{-8} \mathrm{fm}$, to be compared with the typical scattering amplitudes for the nuclear force, which for a range of energies of a few $\mathrm{MeV}$, are of the order of $A_{n}=7 \mathrm{fm}$, although the amplitude can strongly fluctuate with energy as resonances are crossed. It would seem that it is impossible to observe such a tiny gravitational effect, being so small compared to the nuclear amplitude. However, the angular dependence of these amplitudes is quite different. In contrast to the nuclear part, which is essentially independent of the scattering angle for $\theta \ll 1 / L_{\min }$, the gravitational amplitude involves the contribution of a significant number of angular momenta. This in 
turn implies that the gravitational amplitude diverges for small scattering angles. It is the combination of these characteristics which may open a window to observe an interference effect. More specifically, we have derived that $f_{g}(\theta)=K_{0}(\theta / 2)+\delta_{g}(\theta)$, where $K_{0}$ is the Bessel function, which diverges logarithmically as $\theta \rightarrow 0$, and $\delta_{g}(\theta)$ is a smooth function of the angle.

Our analysis shows that at very small scattering angles, the neutron-nucleus differential cross section is given by

$$
\begin{aligned}
\frac{d \sigma}{d \Omega}= & \left|A_{n}(E, \theta)+A_{g}(E, \theta)\right|^{2} \\
\simeq & \left|A_{n}(E, 0)\right|^{2} \\
& +2\left|A_{n}(E, 0)\right| A_{g} \\
& \times \sin \left(\arg \left(A_{n}(E, 0)\right)\right) K_{0}(\theta / 2) .
\end{aligned}
$$

Note that while $\left|A_{n}(E, 0)\right|^{2}$ is about $5 \mathrm{~b} / \mathrm{sr}, 2 \mid A_{n}(E$, $0) \mid A_{g}$ is about $0.4 \mathrm{nb} / \mathrm{sr}$. Discerning such a faint whisper in the midst of the nuclear background roar can be a formidable task. But this feat may be accomplished by carefully monitoring both the angular and energy dependence of the cross section. As the phase of the nuclear amplitude changes as resonances are crossed, the diminutive interference between nuclear and gravitational amplitudes changes from constructive to destructive interference. Our proposal thus requires measurements involving two detectors, one at the smallest possible angles and a second at slightly larger ones. For purely nuclear scattering the ratio of the cross sections (the ratio of detected neutrons) should remain constant as energy is changed. The presence of a gravitational effect of the kind discussed here would be signaled by small fluctuations associated to the interference between the two interactions. Moreover, these fluctuations would not be random, but should correlate with the magnitude of the cross section. A careful analysis of these fluctuations may isolate a gravitational signature.

Considering the progress that has been achieved recently in neutron physics, as shown, for example, in time-of-flight experiments measuring the precession of polarized neutrons through a gas [15], these ideas could be tested in the near future.

Although we have concentrated here on the effects of extra-dimensional gravity on neutron scattering, an entirely different approach can be attempted [16]. The quantum effects of earth's gravity on neutrons have been observed in experiments by Nesvizhevsky et al. [12]. The interference pattern of the neutron density, which is of $\mu \mathrm{m}$ scale, could be affected by deviations from Newton's law at $\mu \mathrm{m}$ scale, produced by the modified gravity of the plates. For $n=2$ the estimate of $R_{\mathrm{c}}$ is in the cm range, so $N \mathrm{D}$ gravity, at the $\mu \mathrm{m}$ range, is $10^{8}$ times larger than normal gravity. The current experimental setup [12] could discern effects for forces $10^{10}$ times larger than normal gravity [16]. Both kinds of dedicated neutron experiments could shed light on the quest for additional dimensions in the universe.

\section{Acknowledgements}

We wish to thank H.G. Börner, R.F. Casten, V. Nesvizhevsky and A. Villari for encouraging discussions. A.F. is supported by CONACyT, Mexico, and J.G.C. by the Spanish DGICyT project FPA200204181-C04-04.

\section{References}

[1] N. Arkani-Hamed, S. Dimopoulos, G. Dvali, Phys. Lett. B 429 (1998) 263;

N. Arkani-Hamed, S. Dimopoulos, G. Dvali, Phys. Rev. D 59 (1999) 086004.

[2] I. Antoniadis, N. Arkani-Hamed, S. Dimopoulos, G. Dvali, Phys. Lett. B 436 (1998) 257.

[3] C.D. Hoyle, et al., Phys. Rev. Lett. 86 (2001) 1418.

[4] L. Randall, R. Sundrum, Phys. Rev. Lett. 83 (1999) 3370; L. Randall, Science 296 (2002) 1422.

[5] J. Blum, et al., Phys. Rev. Lett. 85 (2000) 2426; Z. Chacko, E. Perazzi, hep-ph/0210254.

[6] P. Horava, E. Witten, Nucl. Phys. B 460 (1996) 506; P. Horava, E. Witten, Nucl. Phys. B 475 (1996) 94.

[7] M. Kaku, Quantum Field Theory. A Modern Introduction, Oxford Univ. Press, New York, 1993.

[8] S. Cullen, M. Perelstein, Phys. Rev. Lett. 83 (1999) 268.

[9] D.E. Krause, E. Fischbach, Phys. Rev. Lett. 89 (2002) 190406.

[10] G.F. Giudice, R. Rattazzi, J.D. Wells, Nucl. Phys. B 595 (2001) 250

I. Antoniadis, R. Sturani, Nucl. Phys. B 631 (2002) 66.

[11] J. Byrne, Neutrons, Nuclei and Matter. An Exploration of the Physics of Slow Neutrons, Institute of Physics, Bristol, 1994.

[12] V. Nesvizhevsky, et al., Nature 415 (2002) 297.

[13] Yu.A. Alexandrov, Phys. Part. Nucl. 32 (2001) 708.

[14] A. Villari, et al., Phys. Rev. Lett. 71 (1993) 2551.

[15] A.I. Frank, I.V. Bondarenko, A.V. Kozlov, P. Hoghoj, G. Ehlers, Physica B 297 (2001) 307.

[16] V. Nesvizhevsky, private communication. 